\title{
A Type-2 Fuzzy Scheme for Traffic Density Prediction in Smart City
}

\author{
Sepideh Ravanbakhsh \\ Islamic Azad University \\ Harsin, Iran
}

\author{
Houman Zarrabi \\ Iran ICT Research Center \\ Tehran, Iran
}

\begin{abstract}
There is an increasing demand for using vehicles by growing the population in modern smart cities. This rise has led to traffic jams most of the time, especially during rush hours. In order to tackle this problem different solutions have been proposed in the literature, where each one focuses on a special facet of this problem. In this paper a type-2 fuzzy predictor has introduced so that it estimates the traffic flow in different parts of the city at different times. As a result of that, prevent traffic jams will become avoidable. Also, the impacts of five important parameters that are effective in creation of traffic jams have been studied. These include age pyramid, area population, type of area, the weather, and the day of the week.
\end{abstract}

\section{General Terms}

Intelligent Algorithms, Prediction Systems.

\section{Keywords}

Type-2 Fuzzy Sets, Type-1 Fuzzy Sets, Traffic Prediction, Smart City.

\section{INTRODUCTION}

One of the main difficulties in urban cities is traffic congestion and therefore road commuting has become one of the main concerns for authorities and dwellers. Traffic congestion results into many difficulties such as air pollution, waste of time, resource losses, health problem and over consumption of fuel. So making smart decision can help people to commute comfortably and more energy can be saved. One of the most important criteria in road congestion is traffic flow which depends on lots of parameters, such as weather, time and behavior of drivers. Most of these elements are uncertain in nature and it is not easy to control them. In order to solve these problems, intelligent transportation systems (ITS) have emerged [1] [2].

Many works have been done in different areas related to traffic. For example in cumulative road acoustics has been used in order to identify traffic volume [3]. Some other researchers have performed studies on various prediction models such as Artificial Neural Network models [4] [5] and time series [6] [7]. Other studies have focused on filtering techniques such as Kalman Filter [8]. Moreover, since collecting traffic data and control of its surrounding traffic flow has grabbed lots of attentions, many research have been done in this field and optimization algorithms have used extensively. For example Fuzzy Logic Systems, Neural Network Fuzzy and multi-objective genetic algorithms have been used so far [9] [10] [11] [12]. In [intelligent guardrails], an IoT application called Intelligent Guardrails is introduced and due to detection of traffic state of the roads it utilizes vehicular networks and electronic and mechanical techniques to increase the number of lanes of congested side.
The fuzzy logic is one of the best approaches for designing systems that shows acceptable response against uncertainty [13]. The first one who paid attention to design a fuzzy controller for traffic was Mamdani [1]. A fuzzy controller introduced in [1], [5] in which object detection with tracking algorithm lead to measuring traffic volume.

Unfortunately, type-1 fuzzy logic controllers (FLCs) cannot be used to show uncertainty because they apply accurate type1 fuzzy sets.It has argued that by inhabiting uncertainty in fuzzy sets, it is possible to apply crisp and accurate functions [14]. So, because of lack of enough uncertainty many researchers paid attention to type-2 fuzzy sets. [15]. Geometric fuzzy multi-agent system has proposed in [1],[3]. This approach uses a type- 2 fuzzy inference system. There is a traffic signals rule base and type-2 fuzzy inference system handles this rule base and different level of uncertainty associate with inputs. In [16] general type-2 fuzzy sets and Modified Backtracking Search Algorithm (MBSA) techniques have been proposed in order to control traffic signal scheduling and to reduce waste time. In [2] a hybrid system was suggested which includes a neuro-fuzzy system .Its aim is to predict traffic flow. Simulations showed improvement in the performance measure.

Recent intelligent transport systems use machine vision and recognition algorithms. In [17] a fuzzy logic method has proposed to determine the state of traffic in a road. Road images are obtained from cameras, and vehicles are detected by edge detection and after that their size, distance from neighbor vehicles and vehicle density are calculated and a fuzzy system can be created accordingly. In [1] a smart controller has been introduced. This controller utilizes Fuzzy inference engine and it can control the green light duration based on the traffic density which is calculated by using feature extraction and image subtraction.

\section{FUZZY SETS}

\subsection{Type-1 Fuzzy Sets}

This section introduces Type-1 Fuzzy Sets and Type-2 Fuzzy Sets. An example of T1 fuzzy set A is demonstrated in Fig 1, while only integer numbers are considered in the $\mathrm{x}$ domain. This fuzzy set can be represented as $\{0 / 1,0.5 / 2,0.4 / 3,1 / 4,0.33 / 5,0.2 / 6\}$, where for example $1 / 4$ means that the number 4 in the domain of $A$, has the membership degree of 1 . Membership functions are in different shapes such as Trapezoidal, Triangular, Gaussian and etc. [18]. A sample of Type-1 fuzzy set has been shown in the Fig. 1. 


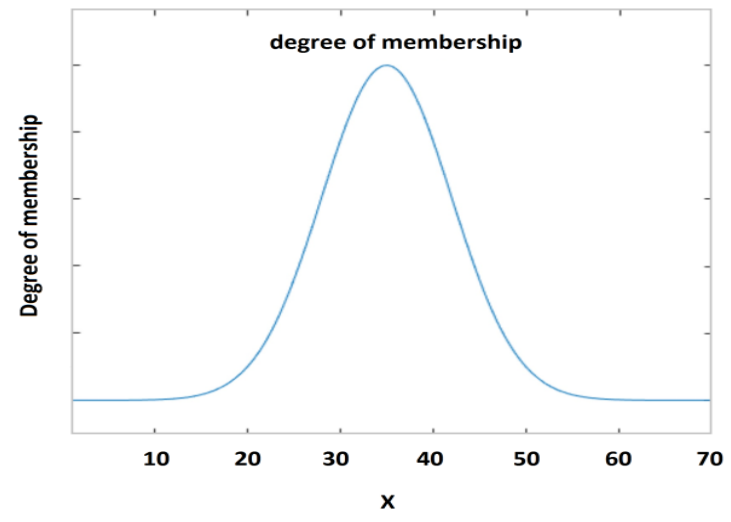

Fig 1: Sample of Type-1 fuzzy membership function

\subsection{Type-2 Fuzzy Sets}

Type-2 fuzzy sets first introduced by Zadeh in 1975 in order to apply uncertainty associated with membership functions themselves. It provides the opportunity to not only use crisp functions but also another fuzzy set. In fuzzy type- 2 there are some values in the membership function $(u)$ for each specific point $(\mathrm{x})$ in the domain. Today there are two categories of type-2 fuzzy sets. First one is general type-2 fuzzy sets in which another fuzzy set uses for the degree of membership function. In other words, fuzzification of $x$ has a membership $\mathrm{u}$ in the primary domain that has a degree of membership called secondary degree of membership. A type-2 fuzzy set $\tilde{A}$ is shown as:

$$
\left\{\left((x, u), \mu_{\tilde{A}}(x, u) \mid \forall x \in X, \forall u \in J_{x} \subseteq[0,1]\right\}\right.
$$

In this equation $0 \leq \mu_{\tilde{A}}(x, u) \leq 1$ and $J_{x} \subseteq[0,1]$ is the primary membership of $\mathrm{x}$ and $\mu_{\tilde{A}}(x, u)$ is the secondary set. Moreover, there is a Footprint Of Uncertainty (FOU) which shows the uncertainty. Second one is interval type-2 fuzzy sets that their secondary degree of membership is always 1 . It means $\mu_{\tilde{A}}(x, u)=1$ and $\forall \mathrm{u} \in \mathrm{J}_{\mathrm{x}} \subseteq[0,1]$ [19] [20]. An interval type-2 fuzzy set [21] $\tilde{A}$ is defined by two membership functions: a lower membership function $\underline{u}_{\widetilde{A}}$ : $X \rightarrow[0,1]$ and an upper membership function $\bar{u}_{\widetilde{A}}: X \rightarrow[0,1]$, where $\underline{u}_{\tilde{A}} \leq \bar{u}_{\tilde{A}}$.[10]. In Fig 2 . A sample of type-2 fuzzy set has been illustrated.

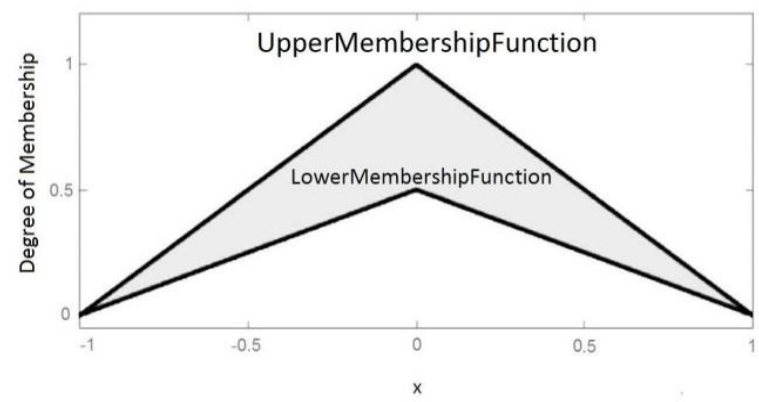

Fig 2: Sample of Type-2 fuzzy membership function

\section{THE PROPOSED SCHEME}

In this paper to predict the traffic density a type-2 fuzzy system is proposed. The most important criteria in creation of congestion are as follows. First, the day: whether it is a workday or weekend has a great impact on traffic. Most of the time on weekends, there is less traffic than working days. Besides, the area itself has a great importance, since it goes without saying that city centers suffers from traffic jams more than suburbs. By the same token, the other vital parameter is weather, which has a great influence on traffic volume. To add to these, it should also be stated that the other affective parameter is population. In can be inferred that that the less populated areas have less traffic density. And last but not least, is age. Areas with younger population have more traffic congestion since students have to go to schools and universities every day and adults have to attend their work as well.

The parameters mentioned above, have been considered as fuzzy system inputs. Each of them has some membership functions. Data has been gathered from experts working in Traffic Department of Kermanshah Province, Iran. That is why, the membership functions are in accordance with reality in this city. For instance, since the city has a moderate weather there are three membership functions, cold, hot and moderate for weather. Also, there are four membership functions for age input which includes: child, teenagers, adults and elderly. In the same way, for day two membership functions have considered, the first one belongs to weekends and the other is dedicated to working days. The output is the traffic volume and an estimate of the velocity a person can drive.

In order to achieve this goal, different membership functions have been used. Gaussian membership functions have been used for weather and age inputs, triangular functions have been applied in area input. Z-shaped and S-shaped membership functions have also used in day and population inputs.

As an example membership functions for temperature and population has demonstrated in Fig.3 and Fig.4 respectively.

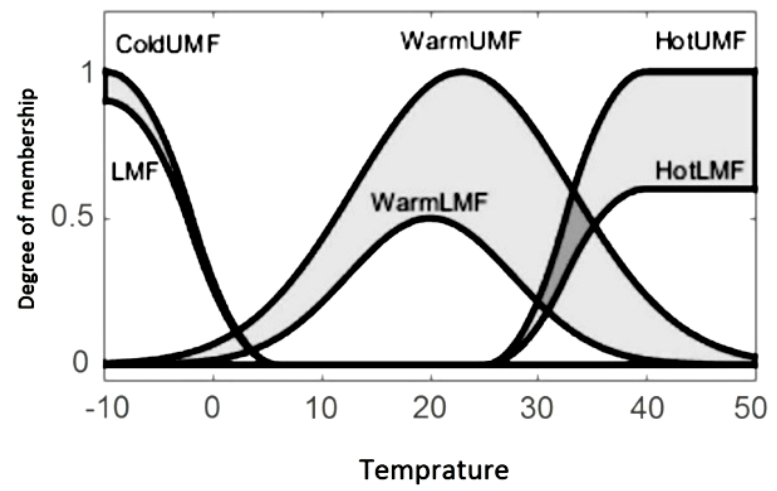

Fig 3: Temperature membership function

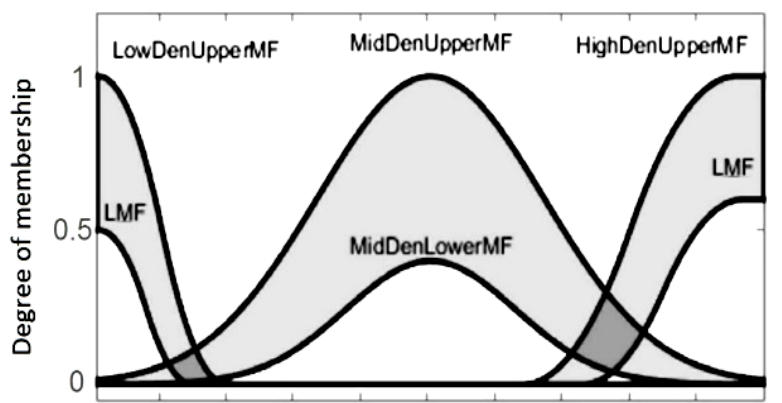

10002000300040005000600070008000900010000 Population

Fig 4: Population membership function 
Table 1: Samples of Rules

\begin{tabular}{|c|c|c|c|c|c|c|}
\hline Rule j & $\mathbf{M F}_{\mathbf{1}}^{\mathbf{j}}$ & $\mathbf{M F}_{\mathbf{2}}^{\mathbf{j}}$ & $\mathbf{M F}_{\mathbf{3}}^{\mathbf{j}}$ & $\mathbf{M F}_{\mathbf{4}}^{\mathbf{j}}$ & $\mathbf{M F}_{\mathbf{5}}^{\mathbf{j}}$ & out $^{\mathbf{j}}$ \\
\hline 1 & Teenager & Highly Populated & Industrial Zone & Weekend & Cold & No Traffic \\
\hline 2 & Teenager & Highly Populated & Industrial Zone & Weekend & Moderate & Low Traffic \\
\hline 3 & Teenager & Highly Populated & Industrial Zone & Weekend & Hot & No Traffic \\
\hline 4 & Adult & Less Populated & Suburb & Weekdays & Cold & Low Traffic \\
\hline 5 & Adult & Less Populated & Suburb & Weekdays & Moderate & Low Traffic \\
\hline 6 & Adult & Less Populated & Suburb & Weekdays & Hot & No Traffic \\
\hline
\end{tabular}

Table 2: Proposed Fuzzy System Prediction Results

\begin{tabular}{|c|c|c|c|c|c|c|}
\hline $\begin{array}{c}\text { Route } \\
\text { Number }\end{array}$ & Area & weather & Age & Population & Day & $\begin{array}{c}\text { System } \\
\text { Prediction }\end{array}$ \\
\hline 1 & City Center & All Weather Conditions & All ages & All populations & Weekdays & $87 \%$ \\
\hline 2 & City Center & All Weather Conditions & All ages & All populations & Weekdays & $84 \%$ \\
\hline 3 & $\begin{array}{c}\text { Suburb and } \\
\text { Industrial zone }\end{array}$ & Moderate & All ages & All populations & All the week & $84 \%$ \\
\hline 4 & $\begin{array}{c}\text { Suburb and } \\
\text { Industrial zone }\end{array}$ & Hot and Cold & All ages & All populations & All the week & $84 \%$ \\
\hline 5 & Residential Area & All Weather Conditions & All ages & All populations & All the week & $81 \%$ \\
\hline
\end{tabular}

A few samples of rules which used by fuzzy system are shown in Table 1 .

\section{EXPERIMENTAL RESULTS}

In order to test the scheme a comparison between results from the proposed fuzzy scheme and the real data from the traffic department have been done and then the accuracy of scheme estimate was calculated. Table 2 shows the estimate of the system for different routes. These estimates have obtained from 1000 samples road observations. Roads have been chosen so that they cover variety of areas, population, weather conditions, etc.

\section{CONCLUSIONS}

In this paper in order to predict the traffic volume in smart cities a Type-2 fuzzy scheme proposed as well as some of the important parameters that have the most impact on traffic congestion. Then the estimates from the proposed fuzzy system compared with the observations obtained from Traffic Department cameras. The simulation result showed that for all the circumstances the proposed system has more than $80 \%$ accuracy and it can be used to help people choose the path with less traffic.

\section{ACKNOWLEDGMENTS}

Thanks to Roja Saketyan who provided authors with traffic data sets and useful insights.

\section{REFERENCES}

[1] Patil R, Srinivasaraghavan A," Smart Traffic Controller using Fuzzy Inference System (STCFIS)", $2^{\text {nd }}$ International Conference on Next Generation Computing Technologies (NGCT-2016) Dehradun, India, October 2016.
[2] Deshpande M, Bajaj R. P, "Short Term Traffic Flow Prediction Based on Neuro-fuzzy Hybrid System", IEEE 2016.

[3] V. Tyagi, S. Kalyanaraman and R. Krishnapuram, "Vehicular Traffic Density State Estimation Based on Cumulative Road Acoustics," in IEEE Transactions on Intelligent Transportation Systems, vol. 13, no. 3, pp. 1156-1166, Sept, 2012.

[4] Stathopoulos, A., Dimitriou, L. Tesekeris, T., "Fuzzy modeling approach for combined forecasting of urban traffic flow", Computer Aided Civil Infrastructure Engineering, 2008.

[5] Vlahogianni, E.I., "Enhancing predictions in signalized arterials with information on short-term traffic flow dynamics”, J. Intelligent Responsive Systems, 2009.

[6] Billings, D., Yang, J.S., "Application of the ARIMA models to urban roadway travel time prediction - a case study", Proc. SMC IEEE Int. Conf., 2006.

[7] Smith, B.L., Williams, B.M., Oswald, R.K., "Comparison of parametric and nonparametric models for traffic flow forecasting”, Transp. Res. C, 2002.

[8] Dehuai Zeng et al.,"Short Term Traffic Flow Prediction Based on Online Learning SVR" Workshop on Power Electronics and Intelligent Transportation System, 2008.

[9] L. A. Klein, "Traffic parameter measurement technology evaluation", Proceedings of the IEEE-IEE vehicle navigation and information systems conference, 1993. 
[10] F. Woelk, S. Gehrig, and R.Koch, "A monocular image based intersectionas- sistant", intelligent vehicles symposium, 2004.

[11] Chen XF, Shi ZK, "A dynamic optimization method for traffic signal timings based on genetic algorithm", J Syst Simul, 2004

[12] X. F. Chen, Z. K. Shi, "Real coded genetic algorithm for signal timing optimization of a single intersection", Proceedings of 2002 international conference on machine learning and cybernetics, vol3, 2002.

[13] Chinyere OU, Francisca OO, Amano OE, "Design and simulation of an intelligent traffic control system", IJAET, 2011.

[14] Mendel J., "Uncertain Rule Based Fuzzy Logic Systems: Introduction and New Directions. Upper Saddle River", NJ: Prentice Hall; 2001.

[15] Zadeh LA. "The concept of a linguistic variable and its application to approximate reasoning”, Inf Sci, 1975.
[16] Khooban M. H., Vafamand N., Liaghat A., Dragicevic T., "An optimal general type-2 fuzzy controller for Urban Traffic Network", ISA Transactions, 2017.

[17] Quiros A. R. F., Bedruz R. A., Uy A. C., Abad A., Bandala A., Dadios E. P., "Machine Vision of Traffic State Estimation Using Fuzzy Logic" IEEE Region 10 Conference (TENCON), 2016.

[18] P. Borkar and L. G. Malik, "Cumulative Acoustic Signal Based Traffic Density State Estimation," Advances in Computing and Communications (ICACC), 2013 Third International Conference on, Cochin, 2013, pp. 169-172.

[19] Schwaab A. A. dos S., Nassar S. M., Freitas Filho de P. J., "Automatic Methods for Generation of Type-1 and Interval Type-2 Fuzzy Membership Functions", Journal of Computer Sciences 2015, 11 (9): 976.987, 2015.

[20] Castillo O.,Melin P., "A review on the design and optimization of interval type-2 fuzzy controllers", Applied Soft Computing 12 (2012) 1267-1278

[21] Runkler Th., Coupland S., John R. "Interval type-2 fuzzy decision making”, International Journal of Approximate Reasoning, 2017. 